\title{
The relationship of plasma transfusion from female and male donors with outcome after cardiac surgery
}

\author{
Ian J. Welsby, MBBS, ${ }^{\text {a }}$ Marla Troughton, MD, ${ }^{\mathrm{b}}$ Barbara Phillips-Bute, $\mathrm{PhD},{ }^{\mathrm{a}}$ Rebecca Ramsey, MS, ${ }^{\mathrm{c}}$ \\ Mary Lee Campbell, MT(ASCP)SBB, ${ }^{\mathrm{d}}$ Nicholas Bandarenko, MD, ${ }^{\mathrm{e}}$ Joseph P. Mathew, MD, ${ }^{\mathrm{a}}$ and \\ Mark Stafford-Smith, MD, ${ }^{\mathrm{a}}$ for members of Cardiothoracic Anesthesiology Research Endeavors ${ }^{\mathrm{f}}$
}

\begin{abstract}
Objective: Pulmonary dysfunction is common in transfused patients recovering from heart surgery. Plasma transfusion from female donors has been linked with rare catastrophic lung injury, but its relationship with outcome after cardiac surgery is poorly understood. We examined whether plasma donor gender is related to postcardiac surgery pulmonary dysfunction and death or prolonged hospitalization.
\end{abstract}

Methods: In this retrospective case-control study, cardiac surgery candidates who received plasma perioperatively from only female donors were compared with male-only recipients who were matched for the number of units transfused and surgery date.

Results: In a dataset of 2157 recipients, there were no blood bank-reported complications, but escalating plasma transfusion was associated with increased 30-day mortality (odds ratio, 1.52 per unit; $P=.0001$ ). From the 1069 recipients receiving plasma exclusively from female or male donors, 390 matched pairs were identified. Recipients of female compared with male donor plasma had a lower incidence of pulmonary dysfunction $(5.9 \%$ vs $10.8 \% ; P=.01)$ and death or hospitalization more than 10 days $(9 \%$ vs $16.4 \% ; P=.002)$ but similar longterm survivals.

Conclusions: Escalating plasma transfusion was associated with 30-day mortality, but female donor plasma recipients had less pulmonary dysfunction and fewer poor outcomes compared with male-only recipients. Although our retrospective study findings neither support nor refute a strategic policy to exclude female donor plasma to reduce catastrophic transfusion-related acute lung injury, they raise concern that such a policy may have unanticipated effects on outcome in patients undergoing cardiac surgery and highlight a need for additional studies in this and other patient groups. (J Thorac Cardiovasc Surg 2010;140:1353-60)

Pulmonary responses to plasma transfusion include circulatory overload, acute lung injury, and dyspnea of unknown cause. ${ }^{1}$ Cardiac surgery is a suitable setting to examine the spectrum of transfusion-related pulmonary dysfunction, because it consumes more than $20 \%$ of transfusions in the United States. Although respiratory risk is rarely considered in the perioperative decision to transfuse patients undergoing heart surgery, their recovery is commonly complicated by pulmonary dysfunction, and adult respiratory distress syndrome (ARDS) occurs after up to $2 \%$ of procedures. $^{2}$ Catastrophic acute lung injury that is directly attributed to

\footnotetext{
From the Department of Anesthesiology, ${ }^{\mathrm{a}}$ Duke University Medical Center, Durham, NC; Department of Pathology, ${ }^{\mathrm{b}}$ University of Alabama, Birmingham, Ala; Carolinas Region American Red Cross, ${ }^{\mathrm{c}}$ Charlotte, NC; Perioperative Services, ${ }^{\mathrm{d}}$ Duke University Medical Center, Durham, NC; Transfusion Medicine, ${ }^{e}$ Duke University Medical Center, Durham, NC; and Cardiothoracic Anesthesiology Research Endeavors, Department of Anesthesiology, ${ }^{\mathrm{f}}$ Duke University Medical Center, Durham, NC.

For members of Cardiothoracic Anesthesiology Research Endeavors, See Appendix 2. Disclosures: None.

Received for publication May 12, 2009; revisions received Sept 16, 2009; accepted for publication Dec 20, 2009; available ahead of print Feb 8, 2010.

Address for reprints: Mark Stafford-Smith, MD, Department of Anesthesiology, Duke University Medical Center, Box 3094 DUMC, Durham, NC 27710 (E-mail: staff002@mc.duke.edu).

0022-5223/\$36.00

Copyright (C) 2010 by The American Association for Thoracic Surgery

doi:10.1016/j.jtcvs.2009.12.035
}

a transfusion is typically indistinguishable from ARDS and, although rarely reported (estimated 200 fatalities per year in the United States), ${ }^{3}$ represents the leading cause of death from hemotherapy. Although such cases are most often related to plasma and occasionally reported in patients undergoing cardiac surgery, of particular interest are the more common but milder nonspecific pulmonary effects that typically go unattributed to transfusion but may also influence outcome. ${ }^{4,5}$ An intensive care unit (ICU) study in which lung injury identified within hours of transfusion in $8 \%$ of patients was associated with subsequent respiratory support needs, extended ICU and hospital stays, and mortality risk highlights the potential for links between plasma transfusion and subsequent hospital course. ${ }^{4}$

Antileukocyte antibodies are more common in women, ${ }^{6}$ presumably because of placental sensitization, and have been linked to cases of transfusion fatalities. ${ }^{1,7}$ In the past several years, this has prompted some national transfusion agencies (eg, the United Kingdom and United States) to restrict the use of female donor plasma on the basis of the mechanism for lung injury attributed to donor antileukocyte antibodies (antihuman leukocyte antigen class I, antihuman leukocyte antigen class II, and antigranulocyte). ${ }^{1,7,8}$ However, the relative importance of leukocyte antibodies compared with other injury mechanisms (eg, nonantibody 


\section{Abbreviations and Acronyms \\ ARDS $=$ adult respiratory distress syndrome \\ ICU = intensive care unit \\ TRALI $=$ transfusion-related acute lung injury}

mediated, "2 hit" hypothesis) and their relevance to the milder pulmonary effects of plasma transfusion are unknown. ${ }^{4}$

Although the need for research into transfusion recipient epidemiology and outcomes is acknowledged, ${ }^{9}$ no studies have yet evaluated the effects of a policy restricting female donor plasma use in patients undergoing cardiac surgery. A single study identifying fresh-frozen plasma transfusion as an independent risk factor for post-cardiac surgery renal failure did not assess donor gender. ${ }^{10}$ To better understand the relationship of donor gender, plasma transfusion, postoperative pulmonary dysfunction, and outcome after cardiac surgery, we assessed Red Cross donor blood bank transfusion and prospectively collected quality assurance data for a cohort of patients undergoing aortocoronary bypass surgery.

\section{MATERIALS AND METHODS}

The patient population included all adult primary, non-emergency, aortocoronary bypass surgery procedures performed at Duke University Medical Center between June 1, 1995, and June 30, 2002; long-term follow-up was to February 28,2007 . Surgery dates were selected from a period preceding concerns regarding donor gender. The primary analysis was performed using a paired subset of patients from those receiving plasma exclusively from female or male donors, matched for the number of plasma units transfused and nearest surgery date using an iterative computer algorithm. The maximum date difference between surgery dates was restricted to 1 year, and no patient was included more than once. Platelets, cryoprecipitate, and other less commonly used blood products containing plasma were not included in the match because pooling of platelet units from multiple donors precluded donor gender assignment.

Standard practice included intraoperative administration of an antifibrinolytic therapy; most often this was $\epsilon$ aminocaproic acid, although because of surgeon preference a few patients instead received aprotinin. The use of cell-saver technology to return washed shed red blood cells to the patient was routine. All plasma units were collected, thawed, and transfused within approved regulatory time guidelines, and plasma transfusion decisions were aided by local guidelines and use of activated clotting time, fibrinogen level, thromboelastograph, prothrombin, and partial thromboplastin time tests. There was a general approach to avoid unnecessary use of blood products. Data on the gender of blood donors were not available to Blood Bank personnel or caregivers. The standard Blood Bank response to a request for plasma is to provide a suitable plasma unit without regard for characteristics other than $\mathrm{ABO}$ compatibility.

We accessed the Duke Databank for Cardiovascular Diseases to obtain patient characteristics and perioperative information. The Databank is prospectively compiled during the hospital stay from contemporaneous medical records, custom datasheets, and laboratory results; quality assurance includes subsequent random chart review for completeness. Survival data were provided by the Duke Clinical Research Institute Follow-up Group. ${ }^{11}$ Only patients receiving plasma units that were successfully cross-referenced with the American Red Cross donor database were included in the study. Computerized cross-referencing between the Carolinas Region American
Red Cross database and the Duke Databank provided donor characteristics for plasma units including gender. A blinded, manual research of Red Cross records for 20 randomly selected plasma units found $100 \%$ data concordance with computerized data including donor gender. Institutional and Red Cross review boards approved data access with patient consent waived for use in the research.

In-hospital complications were recorded using Society of Thoracic Surgeons criteria (see Appendix 1). The a priori defined primary end point was pulmonary dysfunction, a composite of pulmonary edema, pneumonia, and ARDS. Notably, this end point generally evaluates clinically evident pulmonary dysfunction and does not focus on catastrophic transfusion-related acute lung injury (TRALI). A search was also made for voluntary reporting to the Blood Bank of any transfusion reaction that was diagnosed as TRALI by Blood Bank physicians according to the definition at the time. Secondary end points were death within 30 days or prolonged hospital stay ( $>10$ days postoperative $)^{12}$ and long-term survival. Other postoperative complications were recorded, including neurologic (stroke or transient ischemic attack), cardiac (atrial or ventricular arrhythmia, cardiac arrest), infectious (positive blood culture, leg wound infection, sternal wound infection, mediastinitis, or urinary tract infection), and renal (dialysis).

\section{Statistical Analysis}

The matched patient groups were compared for demographic factors and primary and secondary outcomes using generalized estimating equations for paired data and paired $t$ tests for group comparisons of categoric and continuous variables, respectively. Kaplan-Meier log rank test and Cox proportional hazards regression analysis adjusted for the Hannan mortality risk score $^{13}$ were used to evaluate long-term survival.

Additional descriptive analyses included the unmatched dataset of plasma recipients and all plasma units. The ratio of female-to-male plasma donor units was calculated for each recipient and averaged for each complication group. For example, a patient receiving 2 female and 4 male donor plasma units would have a $67 \%$ male donor ratio. For each complication, the female-to-male ratio for the complication group was compared with all other patients using multivariable logistic regression models adjusting for number of plasma units transfused and preoperative Hannan mortality risk score. ${ }^{13,14}$ Statistical analyses were performed using SAS version 8.02 (SAS Inc, Cary, NC).

\section{RESULTS}

During the study period, there were 8743 consecutive primary non-emergency aortocoronary bypass surgery procedures and 6850 plasma units transfused. Of identified plasma units, 6541 (96\%), involving 2157 plasma recipients, were successfully cross-referenced with the American Red Cross donor database. No transfusion reactions from this cohort of patients were reported to the blood supplier for TRALI evaluation. Female donors provided 2917 units (44.6\%), and 1069 patients received plasma from exclusively male or exclusively female donors. In addition to platelets and cryoprecipitate, a small number of other plasma-containing units were transfused, including cryoprecipitate-poor plasma $(n=7)$, plasma frozen within 8 to 24 hours of collection $(\mathrm{n}=17)$, and other plasma-containing units $(\mathrm{n}=37)$.

The 30-day mortality rate for plasma recipients was 132 of $2157(6 \%)$. The number of plasma units transfused was strongly associated with patient mortality risk, with a 1.52-fold increased likelihood of death with each unit (95\% confidence interval, $1.23-1.87 ; P=.0001)$. 
TABLE 1. Patient, procedural, and transfusion characteristics in a matched analysis of patients undergoing aortocoronary bypass surgery receiving plasma from male or female donors

\begin{tabular}{|c|c|c|c|}
\hline & \multicolumn{2}{|c|}{ Transfused plasma } & \multirow[b]{2}{*}{$P$ value } \\
\hline & Female donor only $(n=390)$ & Male donor only $(n=390)$ & \\
\hline \multicolumn{4}{|l|}{ Patient demographic variables } \\
\hline Age, mean (SD), y & $65(10)$ & $66(10)$ & .51 \\
\hline Gender, no. ( $\%$ female $)$ & $102 / 390(26)$ & $144 / 390(37)$ & .002 \\
\hline Height, mean (SD), cm & $170.8(11.1)$ & $169.2(11.3)$ & .12 \\
\hline Weight, mean (SD), kg & $82.7(18)$ & $78.2(17)$ & .001 \\
\hline Ethnicity (\% non-Caucasian) & $35 / 390(9)$ & $54 / 390(14)$ & .03 \\
\hline$\%$ ABO blood types $(\mathrm{O} / \mathrm{A} / \mathrm{B} / \mathrm{AB})$ & $46 / 41 / 9 / 4$ & $43 / 42 / 11 / 4$ & .68 \\
\hline Rhesus blood type $\left(\% \mathrm{Rh}^{+}\right)$ & $338 / 390(87)$ & $348 / 390(89)$ & .27 \\
\hline \multicolumn{4}{|l|}{ Preoperative comorbidities } \\
\hline Diabetes, no. (\%) & $134 / 390(34)$ & $131 / 390(34)$ & .83 \\
\hline Hypertension, no. (\%) & 268/390 (69) & $280 / 390(72)$ & .35 \\
\hline Smoking history, no. $(\%)$ & $155 / 390(40)$ & $160 / 390(41)$ & .7 \\
\hline Previous CHF, no. (\%) & $71 / 390(18)$ & $77 / 390(20)$ & .58 \\
\hline Previous stroke, no. $(\%)$ & $42 / 390(11)$ & $60 / 390(16)$ & .02 \\
\hline Previous MI, no. $(\%)$ & $132 / 390(33)$ & $121 / 390(31)$ & .4 \\
\hline Baseline serum creatinine, mg/dL, mean (SD) & $1.21(0.70)$ & $1.35(1.28)$ & 13 \\
\hline Hannan mortality risk index ${ }^{12}(\mathrm{SD})$ & $0.03(0.05)$ & $0.03(0.04)$ & .36 \\
\hline Charlson comorbidity index ${ }^{13}$ (SD) & $0.86(1.13)$ & $0.88(1.19)$ & .81 \\
\hline \multicolumn{4}{|l|}{ Procedural variables } \\
\hline Preoperative intravenous heparin, no. (\%) & $158 / 374(42)$ & $181 / 367(49)$ & .03 \\
\hline No. of bypass grafts, mean (SD) & $3.3(0.8)$ & $3.3(0.9)$ & .29 \\
\hline CPB duration, min, mean (SD) & $116(39)$ & $119(44)$ & .35 \\
\hline \multicolumn{4}{|l|}{ Transfusion characteristics } \\
\hline Fresh-frozen plasma, no. units, mean (SD) & $1.65(0.76)$ & $1.65(0.76)$ & - \\
\hline No. of units $1 / 2 / 3 />3$ & $189 / 167 / 16 / 18$ & $189 / 167 / 16 / 18$ & - \\
\hline$\%$ donor gender $\mathrm{F} / \mathrm{M}$ & $100 / 0$ & $0 / 100$ & - \\
\hline Platelets no. (SD) & $0.79(0.75)$ & $0.84(0.82)$ & .34 \\
\hline Number of units $0 / 1 / 2 / 3 />3$ & $149 / 182 / 53 / 4 / 2$ & $141 / 188 / 48 / 7 / 6$ & \\
\hline Mean age of units transfused (d) & $3.36(1.10)$ & $3.19(0.08)$ & .13 \\
\hline Packed red blood cells & $3.15(1.27)$ & $3.26(1.21)$ & .18 \\
\hline No. of units $0 / 1 / 2 / 3 />3$ & $27 / 26 / 48 / 51 / 238$ & $29 / 11 / 42 / 54 / 254$ & \\
\hline Mean age of units transfused (d) & $16.47(10.13)$ & $16.95(9.92)$ & .15 \\
\hline Cryoprecipitate no. (SD) & $0.23(0.48)$ & $0.28(0.53)$ & .18 \\
\hline No. of units $0 / 1 / 2 / \geq 3$ & $307 / 74 / 9 / 0$ & 293/84/12/1 & \\
\hline
\end{tabular}

$C H F$, Congestive heart failure; $M I$, myocardial infarction; $C P B$, cardiopulmonary bypass; $S D$, standard deviation.

From the population of 1069 patients receiving exclusively male or female donor plasma, 780 were matched by number of plasma units and the nearest surgery date (390 pairs; Table 1) for inclusion in the primary analysis; the median pair difference in surgery date was 5 days, with a maximum of 343 days. The median follow-up time was 742 days (maximum 2247 days). Fifteen patients in each group received aprotinin. The average age of transfused platelets and red blood cells were similar between the matched groups (Table 1). During the follow-up period, 93 and 109 deaths occurred in the female and male plasma donor groups, respectively.

The likelihood of receiving a platelet transfusion was similar between groups $(P=.44)$, with the majority of patients receiving none. Predicted mortality using the Hannan ${ }^{13}$ risk score was similar between groups $(3.2 \% \pm 0.5 \%$ female vs $3.5 \% \pm 0.4 \%$ male plasma recipients, $P=.36$; Table 1 ).
The primary end point, pulmonary dysfunction (pulmonary edema, pneumonia, or ARDS), was less common in recipients of plasma from female compared with male donors $(5.9 \%$ vs $10.8 \% ; P=.01$, Table 2 , Figure $1, A)$. Female donor plasma recipients were also less likely to have a prolonged postoperative hospital stay or death within 30 days $(9.0 \%$ vs $16.4 \% ; P=.002$, Table 2 , Figure $1, C)$ and had fewer infectious complications $(1 \%$ vs $4 \%, P=.01)$. Adjusted analyses accounting for differences between groups (gender, weight, ethnicity, history of stroke, and preoperative heparin therapy) found similar associations of maleonly plasma with pulmonary dysfunction $(P=.04)$ and death or prolonged hospital stay $(P=.007)$. The relative preponderance of respiratory complications in male-only plasma recipients was consistent when depicted by groups with escalating numbers of plasma units transfused and was 
TABLE 2. Postoperative complications in a matched analysis of patients undergoing aortocoronary bypass surgery receiving plasma from male or female donors

\begin{tabular}{|c|c|c|c|}
\hline & \multicolumn{2}{|c|}{ Transfused plasma } & \multirow[b]{2}{*}{$\begin{array}{c}P \\
\text { value }\end{array}$} \\
\hline & $\begin{array}{l}\text { Female donor } \\
\text { only }(n=390)\end{array}$ & $\begin{array}{c}\text { Male donor } \\
\text { only }(\mathbf{n}=390)\end{array}$ & \\
\hline Pulmonary dysfunction, no. (\%) & $23 / 390(6)$ & $42 / 390(11)$ & $.01 *$ \\
\hline Pulmonary edema, no. (\%) & $22 / 390(6)$ & $32 / 390(8)$ & \\
\hline Pneumonia, no. $(\%)$ & $3 / 390(1)$ & $10 / 390(3)$ & \\
\hline ARDS, no. (\%) & $2 / 390(1)$ & $4 / 390(1)$ & \\
\hline Death or prolonged stay, no. $(\%)$ & $35 / 390(9)$ & $64 / 390(16)$ & $.002 \dagger$ \\
\hline Hospital stay $\geq 10 \mathrm{~d}$, no. $(\%)$ & $26 / 390(7)$ & $52 / 390(13)$ & \\
\hline 30-d mortality, no. (\%) & $11 / 390(3)$ & $18 / 390(5)$ & \\
\hline Cardiac, no. $(\%)$ & $99 / 390(25)$ & $107 / 390(27)$ & .50 \\
\hline Atrial arrhythmia, no. (\%) & $93 / 390(24)$ & $90 / 390(23)$ & \\
\hline Ventricular arrhythmia, no. (\%) & $8 / 390(2)$ & $17 / 390(4)$ & \\
\hline Cardiac arrest, no. $(\%)$ & $4 / 390(1)$ & $6 / 390(2)$ & \\
\hline Neurologic, no. (\%) & $10 / 389(3)$ & $10 / 388(3)$ & .99 \\
\hline Stroke, no. $(\%)$ & $7 / 389(2)$ & $10 / 388(3)$ & \\
\hline $\begin{array}{l}\text { Transient ischemic attack, } \\
\text { no. }(\%)\end{array}$ & $4 / 389(1)$ & $0 / 388(0)$ & \\
\hline Major infection, $\ddagger$ no. $(\%)$ & $3 / 389$ (1) & $14 / 388(4)$ & .01 \\
\hline Positive blood culture, no. (\%) & $1 / 389(0)$ & $5 / 388(1)$ & \\
\hline Leg wound infection, no. (\%) & $1 / 389(0)$ & $4 / 388(1)$ & \\
\hline Sternal wound infection, no. (\%) & $1 / 362(0)$ & 3/364 (2) & \\
\hline Mediastinitis, no. (\%) & $0 / 389(0)$ & $0 / 388(0)$ & \\
\hline \multicolumn{4}{|l|}{ Minor infection } \\
\hline Urinary tract infection, no. $(\%)$ & $8 / 389(2)$ & $11 / 388(3)$ & .47 \\
\hline \multicolumn{4}{|l|}{ Renal } \\
\hline Dialysis, no. $(\%)$ & $4 / 390(1)$ & $5 / 390(1)$ & .74 \\
\hline
\end{tabular}

suggestive of a dose effect (Figure 1, B). Other perioperative complications are presented in Table 2. Kaplan-Meier survival estimates suggest a trend toward longer survival in female compared with male donor plasma recipients (logrank test, $P=.09$ ). However, this effect is not supported by analysis of the hazard ratio adjusted for Hannan score, gender, weight, previous stroke, preoperative heparin therapy, and ethnicity (Caucasian vs non-Caucasian), which is $0.90(95 \%$ confidence interval, $0.67-1.20 ; P=.47)$ (Figure 2).

Secondary analyses in the larger population of all plasma recipients $(\mathrm{n}=2157)$, to compare donor ratios (female-tomale) in patients with and without complications, supported concerns regarding adverse outcomes associated with transfusion of male donor plasma from our primary findings (Table 3). There was a higher likelihood of receiving plasma from a male donor in patients with pulmonary dysfunction $(\mathrm{n}=201,60.9 \%$ vs $54.8 \%, P=.03)$ and prolonged hospital stay or death within 30 days $(\mathrm{n}=366,59.1 \%$ vs $54.6 \%$, $P=.02)$ compared with uncomplicated patients. Notably,
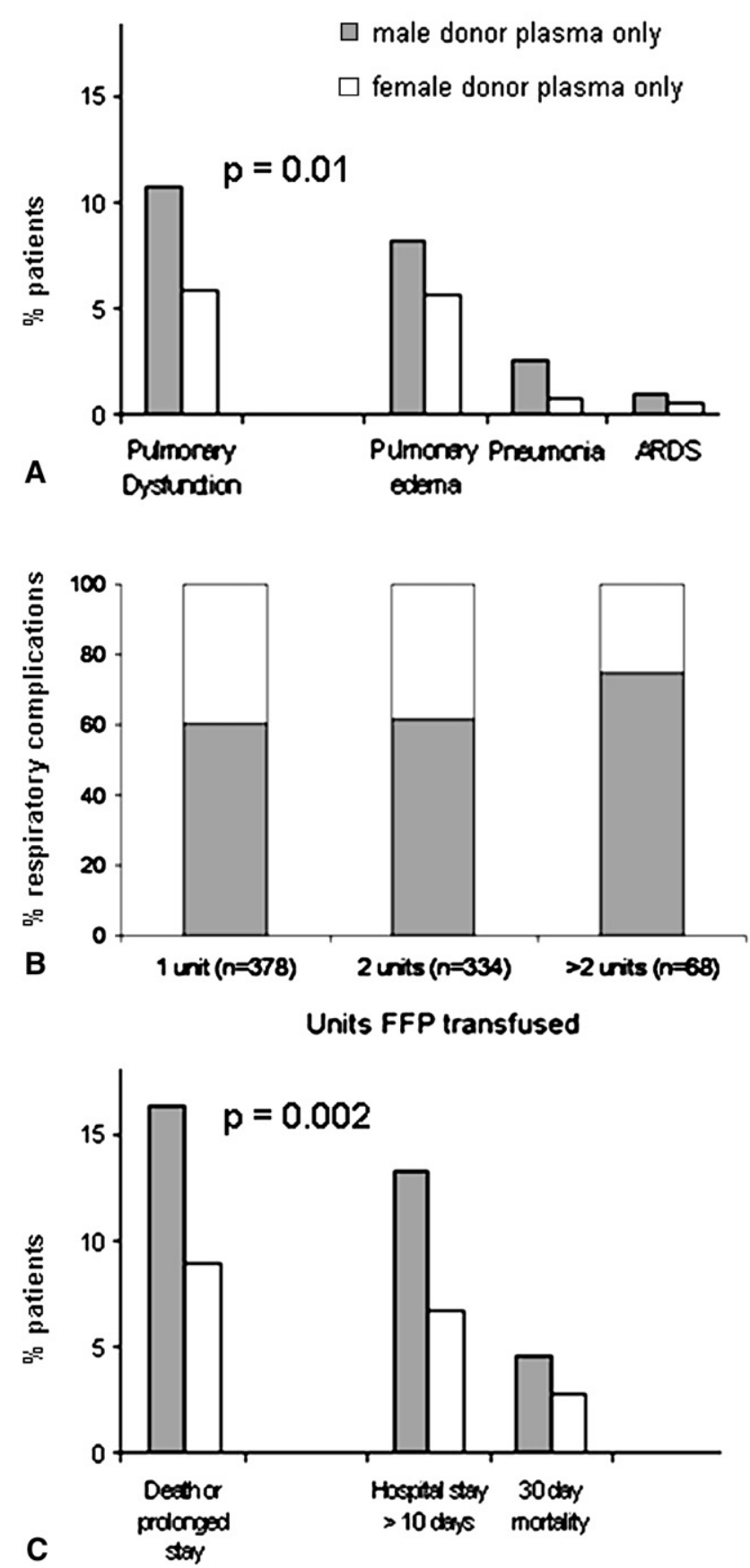

FIGURE 1. Postoperative pulmonary dysfunction rates in matched patients undergoing aortocoronary bypass surgery grouped by contributing types of complications (A) and the number of units they received (B) $(1,2$, or $>2$ units) reflect an excess of pulmonary complications in equivalent recipients of male relative to female donor plasma. Major complications (30-day mortality or hospitalization $>10$ days) are also more common in equivalent recipients of male relative to female donor plasma (C).

the pattern of increased major infectious complications in male-only recipients noted in our matched analysis was not present in this analysis. Other postoperative complications are presented in Table 3. 


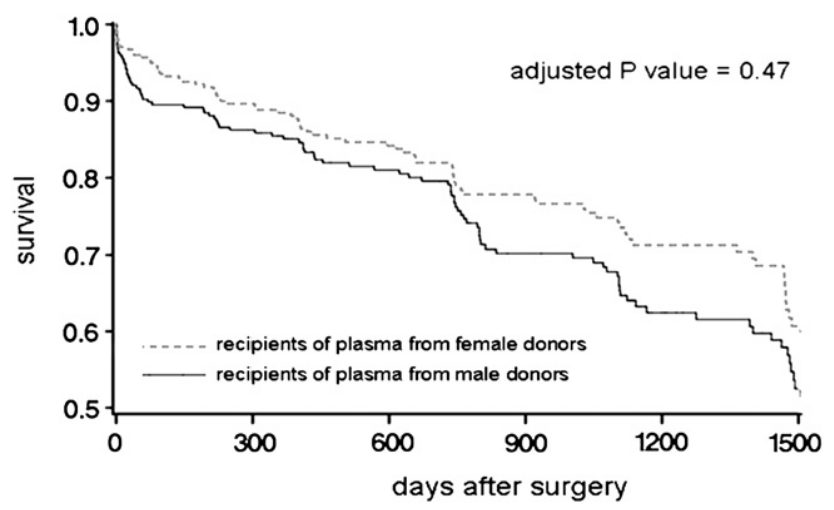

FIGURE 2. Kaplan-Meier estimates of survival in recipients of female compared with male donor plasma, log-rank test $P=.09$. Hazard ratio for female donor plasma, as estimated by the Cox proportional hazards model, adjusted for Hannan score, gender, weight, ethnicity (Caucasian vs non-Caucasian), previous stroke, and preoperative heparin therapy is $0.90 ; 95 \%$ confidence interval, $0.67-1.20 ; P=.47$.

\section{DISCUSSION}

Although pulmonary dysfunction was common $(8 \%)$ and strongly associated with plasma transfusion in our study of patients undergoing cardiac surgery, we found no link between female donor plasma and increased complications. In fact, plasma from male donors was associated with worse outcome. Matched analysis of female- and male-only donor plasma recipients revealed less pulmonary dysfunction and fewer poor outcomes (hospitalization $>10$ days or death within 30 days), but no difference in long-term survival for recipients of female donor plasma. In addition, our primary findings were supported by a consistent excess of pulmonary complications in male compared with female donor plasma recipients when patients were grouped by number of plasma units received in a pattern suggestive of a dose effect. Finally, a secondary assessment of the larger unmatched sample of all patients receiving plasma from male or female donors also supported our primary findings of less pulmonary dysfunction and fewer poor outcomes with female donor plasma. Our study is not designed to evaluate catastrophic TRALI episodes but raises concern that any reduction in this rare condition related to policies restricting female donor plasma use may have important unrecognized adverse consequences in patients undergoing cardiac surgery.

Reductions in reported deaths related to catastrophic TRALI that may be a consequence of "male only" plasma policies are good news but do not reflect overall outcomes (including deaths from other causes) in large patient groups. Agencies reviewing methods to assess changes in transfusion policy describe epidemiologic studies as a "timely and highly desirable", analytic approach despite their potential for bias and confounding. ${ }^{9}$ Support from a broad assessment of outcomes is needed to endorse the implementation of transfusion policies restricting female donor plasma use,
TABLE 3. Data including all 2157 aortocoronary bypass surgeries comparing the likelihood of a transfused plasma unit coming from a female donor in patients who did or did not have respiratory and other postoperative complications

\begin{tabular}{|c|c|c|c|}
\hline $\begin{array}{c}\text { Total population } \\
\quad \mathbf{n}=\mathbf{2 1 5 7}\end{array}$ & $\begin{array}{c}\text { Complication } \\
(\% \text { plasma units } \\
\text { from female donor })\end{array}$ & $\begin{array}{c}\text { No complication } \\
(\% \text { plasma units } \\
\text { from female donors })\end{array}$ & $\begin{array}{c}P \\
\text { value } \\
\end{array}$ \\
\hline \multicolumn{4}{|l|}{ Complication category } \\
\hline Respiratory $(\mathrm{n}=201)$ & 39.1 & 45.2 & .03 \\
\hline $\begin{array}{l}\text { Pulmonary edema } \\
\qquad(\mathrm{n}=159)\end{array}$ & 37.3 & 44.9 & \\
\hline Pneumonia $(\mathrm{n}=57)$ & 44.4 & 44.2 & \\
\hline $\operatorname{ARDS}(\mathrm{n}=19)$ & 44.1 & 44.2 & \\
\hline $\begin{array}{l}\text { Death or prolonged } \\
\text { stay }(\mathrm{n}=366)\end{array}$ & 40.9 & 45.4 & .02 \\
\hline $\begin{array}{l}\text { Hospital stay } \geq 10 \mathrm{~d} \\
\quad(\mathrm{n}=279)\end{array}$ & 40.7 & 45.2 & \\
\hline $\begin{array}{l}\text { 30-d mortality } \\
\quad(\mathrm{n}=132)\end{array}$ & 41.7 & 44.8 & \\
\hline Cardiac $(n=467)$ & 44.7 & 44.6 & .97 \\
\hline Neurologic $(n=138)$ & 41.7 & 44.8 & .34 \\
\hline $\begin{array}{l}\text { Serious infection* } \\
\quad(\mathrm{n}=84)\end{array}$ & 41.6 & 44.7 & .46 \\
\hline $\begin{array}{l}\text { Acute kidney injury } \dagger \\
\quad(\mathrm{n}=308)\end{array}$ & 46.9 & 43.4 & .15 \\
\hline
\end{tabular}

a policy that was developed primarily from "circumstantial" 3 serologic evidence (linking leukocyte antibodies with fatal lung injury/TRALI). ${ }^{6}$ One explanation for our findings could be that patients undergoing cardiac surgery are a special case with regard to "male only" plasma and complication rates, but large outcome studies in other patient groups will be required to explore whether our findings are isolated or spurious.

Lung injury observed within hours of plasma transfusion in critically ill patients is common $(8 \%-10 \%)$ and highly associated with poor outcome. ${ }^{15}$ Although no reports have examined donor gender with regard to lung function in cardiac surgery cohorts, 3 studies exist in critically ill patients. ${ }^{4,16,17}$ As in our study, each report confirms strong associations between escalating plasma use and lung injury, but there is no consistent pattern with regard to donor gender. An ICU investigation similar in design to the current study compared 224 paired plasma recipients ( $\geq 3$ units), matched for donor gender, and found no difference in lung injury but more ventilator-free days (28 vs $27 ; P=.006$ ) in male-only recipients. ${ }^{17} \mathrm{~A}$ second report from the same group identified more lung injury in 901 medical ICU patients with female compared with male donor plasma (odds ratio, 5.09 vs 1.60). ${ }^{4}$ Finally, in a British cohort of 
211 consecutive ruptured aortic aneurysm surgeries over an 8 -year period, a blood supply change to male-only plasma was associated with a decrease in lung injury $(36 \%$ vs $21 \% ; P=.04)$ but no change in time to tracheal extubation or 30-day survival. ${ }^{16}$ Unfortunately, transfusion policy changes during this last study are difficult to separate from other diagnostic and surgical practice changes during the same period. In our study of patients undergoing cardiac surgery, we found less pulmonary dysfunction and better outcome (less prolonged hospitalization or death within 30 days) but no difference in long-term survival in recipients of female donor plasma. Collectively, these investigations provide insufficient consensus to support simple understanding of the relationship among plasma transfusion, donor gender, pulmonary dysfunction, and outcome in critically ill patients.

Our study has limitations, including lack of power to evaluate rare catastrophic TRALI; however, the sample size of our study is more than sufficient to study short- and longterm postoperative outcome and the more common perioperative pulmonary dysfunction presumed to be the consequence of cumulated less extreme pulmonary insults. Although our data do not allow for assessment of timing between plasma transfusion and the onset of pulmonary dysfunction or the existence of pulmonary overload or leukocyte antibody status, even if details of these events were known, disentangling the relative importance of numerous potential contributors to a burden of pulmonary dysfunction is difficult. For example, postoperative plasma transfusion may compound an already established cardiopulmonary bypass-related ischemic lung insult, without clear evidence of synchronous timing between transfusion and pulmonary dysfunction. The majority of plasma administration to patients undergoing cardiac surgery occurs early postoperatively to treat coagulopathy, and tracheal extubation typically occurs within hours of surgery and is contingent on cessation of bleeding; thus, recognition of significant pulmonary dysfunction is likely to follow plasma transfusion in almost every case.

Aprotinin use and transfusion of other plasma-containing products (eg, platelets, cryoprecipitate) was not accounted for in our analysis but occurred infrequently and at similar rates between groups. Adjustment for patient characteristics that differed between groups in our male versus female-only plasma recipient analysis (Table 1) did not eliminate the observed association of male-only plasma with pulmonary dysfunction $(P=.04)$ and death or prolonged hospital stay $(P=.007)$. Ideally, our analysis would have included the pregnancy history of female plasma donors and the gender of transfused platelet units (especially those apheresis units with high plasma volume); unfortunately, parity data were not available, and platelet doses at our institution are often pooled from multiple donors. However, our study does closely simulate the current policy regarding plasma that excludes female donor plasma regardless of donor parity. Strengths of our analysis compared with previous studies include assessment of a large, well-characterized population involving long-term follow-up and the relative reproducibility of the physiologic stress of elective cardiac surgery compared with previous studies involving heterogeneous populations of critically ill patients and patients undergoing emergency surgery.

Interpreting our study findings requires speculation on a plausible biologic foundation. Clinical data from studies, such as Palfi and colleagues, ${ }^{18}$ highlights the rationale for limiting the use of female donor plasma. In a randomized double-blind crossover study, these authors compared inflammatory mediators and oxygenation in 100 critically ill patients with transfusion of plasma from multiparous versus non-multiparous donors. Multiparous plasma was associated with poorer oxygenation, higher tumor necrosis factor- $\alpha$ levels, and 4 (vs 1) transfusion reactions. The pathophysiology presumed to be operative in Palfi and colleagues' study involves leukocyte antibody-mediated mechanisms. Leukocyte antibodies are most common in multiparous women $(25 \%)$ but are often detected in nulliparous women $(8 \%){ }^{19}$ However, constituents other than leukocyte antibodies have also been implicated in plasmarelated lung injury through effects involving modification or activation of inflammation (eg, cytokines, soluble CD40 ligand, and lysophosphatidylcholine).,5,20,21 Although female donor leukocyte antibodies may be the most important mediator of catastrophic TRALI, ${ }^{22,23}$ the relative significance of this and alternate mechanisms in mediating other harmful effects of plasma transfusion are largely unknown.

Profound proinflammatory stimuli, such as cardiopulmonary bypass, ${ }^{20}$ are an unavoidable aspect of cardiac surgery that may influence the effects of harmful mediators in transfused plasma. Although inflammatory mediators have not been investigated with regard to gender-related differences in stored plasma, cytokine effects in men have been linked with excess postoperative pulmonary dysfunction. ${ }^{24,25}$ It is plausible that lung dysfunction mediated by leukocyte antibodies may be the most easily recognized (ie, lethal) mechanism, ${ }^{22,23}$ but this does not prevent injury mediated by other plasma-related mechanisms being cumulatively equivalent or more important. ${ }^{5}$

Although our data are intriguing, they neither support nor refute blood systems decisions to limit the use of female plasma to reduce TRALI. However, our study is one of only a few to assess outcomes related to this policy change and does provide retrospective data that expose potentially concerning effects that suggest that such a change may have an impact on outcomes other than TRALI in patients undergoing cardiac surgery. Patients undergoing cardiac surgery are a highly monitored well-documented and sizeable subset of high-risk plasma recipients who may represent an ideal patient group for ongoing assessment of changes in 
transfusion policy. Although our study involves data from before the implementation of a "male only" plasma policy, in this case the policy being assessed has already been implemented. However, future assessment of changes in blood management could occur before their clinical introduction.

The few cohort studies investigating changes in the plasma supply and outcome do not yet provide an endorsement for the policy of restricting use of female donor plasma. Continuing study of this problem will also require agreement on the most relevant measure of benefit and improved patient care, including whether blood bank-reported catastrophic TRALI, or more common pulmonary events that meet the standard definition of TRALI, are satisfactory or sufficient end points to measure success. ${ }^{1,5,26}$

\section{CONCLUSIONS}

Our matched analysis of transfused patients undergoing aortocoronary bypass surgery found more pulmonary dysfunction and an increased risk of prolonged hospital stay or death in those receiving exclusively male compared with female donor plasma. These observations do not support exclusion of female donor plasma as a method to improve outcome or reduce postoperative pulmonary dysfunction for transfused patients undergoing cardiac surgery. Outcome studies are an essential part of assessment of any change in practice, including the supply of blood components. Confirmation of our findings and those of similar studies of critically ill patients should be sought in analyses of additional large datasets and randomized trials.

\section{References}

1. Toy P, Popovsky MA, Abraham E, Ambruso DR, Holness LG, Kopko PM, et al. Transfusion-related acute lung injury: definition and review. Crit Care Med. 2005;33:721-6.

2. Asimakopoulos G, Smith PL, Ratnatunga CP, Taylor KM. Lung injury and acute respiratory distress syndrome after cardiopulmonary bypass. Ann Thorac Surg. 1999;68:1107-15.

3. Strong M, Shoos-Lipton K. Transfusion-related acute lung injury. Association Bulletin \#06-07 (November 3, 2006). Bethesda, MD: AABB; 2006.

4. Gajic O, Rana R, Winters JL, Yilmaz M, Mendez JL, Rickman OB, et al. Transfusion-related acute lung injury in the critically ill: prospective nested case-control study. Am J Respir Crit Care Med. 2007;176:886-91.

5. Davis A, Mandal R, Johnson M, Makar R, Stowell C, Dzik S. A touch of TRALI. Transfusion. 2008;48:541-5.

6. Maslanka K, Michur H, Zupanska B, Uhrynowska M, Nowak J. Leucocyte antibodies in blood donors and a look back on recipients of their blood components. Vox Sang. 2007;92:247-9.
7. Kopko PM, Paglieroni TG, Popovsky MA, Muto KN, MacKenzie MR, Holland PV. TRALI: correlation of antigen-antibody and monocyte activation in donor-recipient pairs. Transfusion. 2003;43:177-84.

8. Bux J. Transfusion-related acute lung injury (TRALI): a serious adverse event of blood transfusion. Vox Sang. 2005;89:1-10.

9. Hillyer CD, Blumberg N, Glynn SA, Ness PM. Transfusion recipient epidemiology and outcomes research: possibilities for the future. Transfusion. 2008;48: 1530-7.

10. Mangano DT, Tudor IC, Dietzel C. The risk associated with aprotinin in cardiac surgery. N Engl J Med. 2006;354:353-65.

11. Shaw AD, Stafford-Smith M, White WD, Phillips-Bute B, Swaminathan M, Milano $\mathrm{C}$, et al. The effect of aprotinin on outcome after coronary-artery bypass grafting. N Engl J Med. 2008;358:784-93.

12. Bennett-Guerrero E, Slaughter TF, White WD, Welsby IJ, Greenberg CS, ElMoalem $\mathrm{H}$, et al. Preoperative anti-PF4/heparin antibody level predicts adverse outcome after cardiac surgery. J Thorac Cardiovasc Surg. 2005;130:1567-72.

13. Hannan E, Kilburn H, Racz M, Shileds E, Chassin M. Improving the outcomes of coronary artery bypass surgery in New York State. JAMA. 1994;271:761-6.

14. Charlson ME, Pompei P, Ales KL, MacKenzie CR. A new method of classifying prognostic comorbidity in longitudinal studies: development and validation. J Chronic Dis. 1987;40:373-83.

15. Khan H, Belsher J, Yilmaz M, Afessa B, Winters JL, Moore SB, et al. Fresh-frozen plasma and platelet transfusions are associated with development of acute lung injury in critically ill medical patients. Chest. 2007;131:1308-14.

16. Wright SE, Snowden CP, Athey SC, Leaver AA, Clarkson JM, Chapman CE et al. Acute lung injury after ruptured abdominal aortic aneurysm repair: the effect of excluding donations from females from the production of fresh frozen plasma. Crit Care Med. 2008;36:1796-802.

17. Gajic O, Yilmaz M, Iscimen R, Kor DJ, Winters JL, Moore SB, et al. Transfusion from male-only versus female donors in critically ill recipients of high plasma volume components. Crit Care Med. 2007;35:1645-8.

18. Palfi M, Berg S, Ernerudh J, Berlin G, Palfi M, Berg S, et al. A randomized controlled trial of transfusion-related acute lung injury: is plasma from multiparous blood donors dangerous? Transfusion. 2001;41:317-22.

19. Densmore TL, Goodnough LT, Ali S, Dynis M, Chaplin H. Prevalence of HLA sensitization in female apheresis donors. Transfusion. 1999;39:103-6.

20. Silliman CC, Elzi DJ, Ambruso DR, Musters RJ, Hamiel C, Harbeck RJ, et al. Lysophosphatidylcholines prime the NADPH oxidase and stimulate multiple neutrophil functions through changes in cytosolic calcium. J Leukoc Biol. 2003;73: 511-24.

21. Khan SY, Kelher MR, Heal JM, Blumberg N, Boshkov LK, Phipps R, et al. Soluble CD40 ligand accumulates in stored blood components, primes neutrophils through CD40, and is a potential cofactor in the development of transfusionrelated acute lung injury. Blood. 2006;108:2455-62.

22. Eder AF, Herron R, Strupp A, Dy B, Notari EP, Chambers LA, et al. Transfusionrelated acute lung injury surveillance (2003-2005) and the potential impact of the selective use of plasma from male donors in the American Red Cross. Transfusion. 2007; 47:599-607.

23. Stainsby D, Jones H, Asher D, Atterbury C, Boncinelli A, Brant L, et al. Serious hazards of transfusion: a decade of hemovigilance in the UK. Transfus Med Rev. 2006;20:273-82.

24. Trotter A, Muck K, Grill HJ, Schirmer U, Hannekum A, Lang D. Gender-related plasma levels of progesterone, interleukin- 8 and interleukin-10 during and after cardiopulmonary bypass in infants and children. Crit Care. 2001;5:343-8.

25. Seghaye MC, Qing M, von Bernuth G. Systemic inflammatory response to cardiac surgery: does female sex really protect? Crit Care. 2001;5:280-2.

26. Kleinman S, Caulfield T, Chan P, Davenport R, McFarland J, McPhedran S, et al. Toward an understanding of transfusion-related acute lung injury: statement of a consensus panel. Transfusion. 2004;44:1774-89. 
APPENDIX 1. Society of Thoracic Surgery and Duke Databank for Cardiovascular Diseases definitions of postcardiac surgery complications

Respiratory

Pulmonary edema

Pneumonia

ARDS

Cardiac

Atrial or ventricular arrhythmia

Cardiac arrest

Neurologic

Stroke

Transient ischemic attack

Infectious

Positive blood culture

Leg wound infection

Sternal wound infection

Mediastinitis
Dyspnea, rales, and radiographic findings compatible with edema

Radiographic infiltrate and growth of typical respiratory pathogen or heavy growth of atypical respiratory pathogen arterial oxygen tension $<50 \mathrm{~mm} \mathrm{Hg}$ despite inspired oxygen fraction $>0.5$ or pulmonary capillary wedge pressure

$<18 \mathrm{~mm} \mathrm{Hg}$

Arrhythmia requiring cardioversion or medications

Advanced cardiac life support in the postoperative period

Loss of neurologic function by ischemia abrupt onset lasting $>24 \mathrm{~h}$ with residual neurologic signs

Loss of neurologic function by ischemia abrupt onset lasting for $<24 \mathrm{~h}$

Positive blood culture in the postoperative period

Incisional infection requiring dressings and intravenous antibiotics

Incisional infection requiring dressings and intravenous antibiotics

Documented infection without return to operating room

\section{APPENDIX 2. Members of the Cardiothoracic Anesthesiology Research Endeavors (C.A.R.E.), Department of Anesthesiology, Duke University Medical Center}

Director: Joseph P. Mathew, MD

Anesthesiology: Solomon Aronson, MD, Katherine P. Grichnik, MD, Steven Hill, MD, G. Burkhard Mackensen, MD, PhD, Joseph P. Mathew, MD, Mark F. Newman, MD, Barbara Phillips-Bute, PhD, Mihai V. Podgoreanu, MD, Andrew D. Shaw, MD, Mark Stafford-Smith, MD, Madhav Swaminathan, MD, Ian Welsby, MD, William D. White, MPH, Lisa Anderson, Lauren Baker, BS, Jerry Dove, RN, Bonita L. Funk, RN, Roger L. Hall, AAS, Gladwell Mbochi, AAS, Tiffany Bisanar, RN, Prometheus T. Solon, MD, Peter Waweru

Perfusion Services: Kevin Collins, BS, CCP, Greg Smigla, BS, CCP, Ian Shearer, BS, CCP

Surgery: Thomas A. D'Amico, MD, R. Duane Davis, MD, Donald D. Glower, MD, R. David Harpole, MD, G. Chad Hughes, MD, James Jaggers, MD, Shu Lin, MD, Andrew Lodge, MD, James E. Lowe, MD, Carmelo Milano, MD, Peter K. Smith, MD, Jeffrey Gaca, MD, Mark Onatis, MD

Cardiology: Eric Peterson, MD, MPH 